\title{
VALORES DE EFECTO RESERVORIO MARINO PARA LOS ÚLTIMOS 5.000 AÑOS OBTENIDOS EN CONCHEROS DE LA COSTA ATLÁNTICA NORPATAGÓNICA (GOLFO SAN MATÍAS, ARGENTINA)
}

CRISTIAN M. FAVIER DUBOIS

\section{RESUMEN}

A fin de evaluar el efecto reservorio marino -ERM- en moluscos de la costa norte del golfo San Matías (Patagonia, Argentina) se dataron por acelerador (AMS) diez pares valva-carbón procedentes de concheros antrópicos ubicados a lo largo de ese litoral. Las diferencias de edad obtenidas fluctúan entre 205 y 358 años radiocarbónicos para el período comprendido entre ca. 5.300 y ca. 700 años AP, no registrándose tendencias particulares a lo largo de ese intervalo. Factores locales como la conexión restringida con el mar abierto o la presencia de carbonatos envejecidos en la faja intermareal no han mostrado un efecto visible en los resultados alcanzados, aunque se ha detectado un posible efecto de edad del material en relación con algunos arbustos utilizados como leña. El promedio de las diferencias de edad, atribuibles al efecto reservorio marino, es de $266 \pm 51$ años. Cabe destacar que este valor local resulta sensiblemente menor al oceánico global de referencia, y representaría una edad de ajuste válida para la corrección de fechados sobre valvas en los numerosos sitios arqueológicos presentes en ese litoral desde el Holoceno medio.

PALABRAS CLAVE: efecto reservorio marino, moluscos arqueológicos, norpatagonia, Holoceno medio-tardío.

\section{MARINE RESERVOIR EFFECT VALUES FOR THE LAST 5.000 YEARS FROM SHELL- MIDDENS LOCATED IN THE ATLANTIC NORTH PATAGONIAN COAST (SAN MATÍAS GULF, ARGENTINA)}

\footnotetext{
ABSTRACT

Ten shell-charcoal pairs obtained from archaeological shell-middens along the San Matías gulf (Northern Patagonia, Argentina) were dated by accelerator (AMS) in order to evaluate the local marine reservoir effect -MRE-. The age differences obtained fluctuate between 205 and 358 radiocarbon years from ca. 5.300 to $c a$. 700 yr BP, not recording any temporal tendency in such time span. Local factors as restricted connection with the open sea or the presence of aged carbonates do not show a visible 
influence on this effect along the gulf coastline. However it has been detected a possible age offset in relation with the age at death of some shrubs used as fuel. The mean value of age differences, related to reservoir effect, was $266 \pm 51$ yr. It may be highlighted that this local value is perceptibly lower than the standard mean oceanic one, and would represent a useful age for correcting radiocarbon dates in shells from the abundant archaeological sites present in this area since Middle Holocene times.

KEY WORDS: marine reservoir effect, archaeological mollusks, Northern Patagonia, Middle-Late Holocene.

\section{INTRODUCCIÓN Y OBJETIVOS}

El golfo San Matías es el más septentrional de la costa atlántica patagónica, y uno de los de mayores dimensiones, con $150 \mathrm{~km}$ de largo (entre $40^{\circ} 45^{\prime}$ y $42^{\circ} 15^{\prime}$ de latitud S) y $140 \mathrm{~km}$ de ancho (de 65ำ a 63ำ de longitud $\mathrm{O}$ aproximadamente). La región se caracteriza por poseer un clima semiárido con una temperatura media de unos $15^{\circ} \mathrm{C}$ y precipitaciones entre 200 y 300 mm. Su vegetación corresponde a la provincia fitogeográfica del Monte, con predominancia de comunidades arbustivas dominadas por Larrea sp. (Cabrera y Willink 1980; Bran et al. 1985).

En el año 2004 se iniciaron investigaciones arqueológicas sistemáticas en la costa oeste y norte de este golfo, litoral que corresponde a la Provincia de Río Negro (Fig. 1). Este relevamiento mostró particularmente en la costa norte la conspicua presencia de acumulaciones de valvas antrópicas (denominadas concheros o conchales) correspondientes al Holoceno medio y tardío, asociadas con numerosa evidencia arqueofaunística y artefactual. (Favier Dubois et al. 2006). Estas acumulaciones corresponden a eventos de consumo de moluscos mitílidos, resultando notable la gran proporción de valvas enteras que contienen, así como la frecuente presencia de fragmentos de carbón entre ellas (Favier Dubois y Borella 2007). La ubicua representación de estos concheros en el espacio y el tiempo así como la estrecha asociación entre valvas y carbones en los mismos alentó la implementación de estudios de efecto reservorio marino (ERM). Este efecto es resultado de la diferente actividad radiocarbónica que presentan los reservorios marino y atmosférico, así como los organismos en equilibrio con ellos, y se expresa como una "edad aparente" que tiende a "envejecer" a los organismos marinos respecto a los continentales (Olsson 1983; Figini 1999). Este efecto varía en función de la latitud (es mínimo en el ecuador y máximo en regiones polares) y de diversos factores locales, pudiendo asimismo variar a lo largo del tiempo en una misma región (Kennett et al. 1997; Deo et al. 2004).

En el marco de un programa de dataciones tendiente a potenciar el uso de indicadores cronológicos para datar los abundantes concheros del litoral bajo estudio, los objetivos de este trabajo fueron:

- obtener datos acerca de la variabilidad del efecto reservorio sobre moluscos en tiempo y espacio a lo largo de la costa norte del golfo San Matías;

- evaluar el efecto de factores locales como la morfología costera, la presencia de carbonatos envejecidos o el aporte de aguas continentales en las diferencias de edad obtenidas;

- obtener un valor de ERM promedio que permita ser utilizado a futuro para la corrección de edades radiocarbónicas sobre valvas de mitílidos procedentes de este litoral.

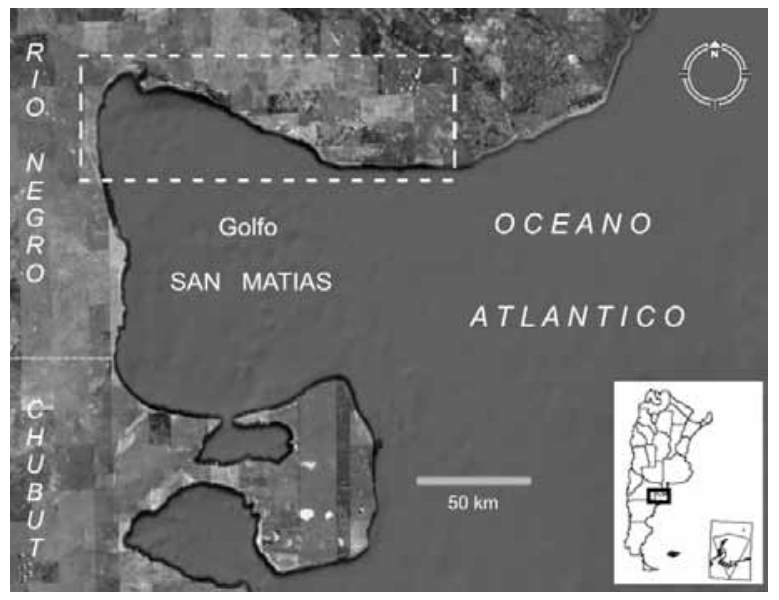

Fig. 1. Imagen satelital del golfo San Matías en el litoral norpatagónico. El área bajo estudio se observa punteada (imagen base tomada de Google Earth 2009). 
Como antecedente de este tipo de estudios en la costa atlántica patagónico-fueguina pueden mencionarse los trabajos realizados en sitios del Canal de Beagle (Tierra del Fuego, Argentina). Allí se obtuvieron edades de ERM de 620 140 años (Albero et al. 1986) en referencia a un promedio entre distintas especies marinas, y de 556+61 años (Albero et al. 1987) en relación con mejillones. Otra investigación, orientada en este caso a explorar la variabilidad actual del efecto reservorio marino, fue desarrollada en localidades costeras de Patagonia y Tierra del Fuego por Cordero et al. (1999 y 2003). Este trabajo mostró importantes diferencias de edad atribuibles al efecto reservorio generadas por factores locales en diferentes puntos de ese extenso litoral, pero no incluyó muestras de la costa rionegrina.

\section{MATERIALES Y MÉTODOS}

Para esta primera aproximación al estudio del efecto reservorio marino en la costa norte del golfo San Matías, se seleccionaron pares valva-carbón correspondientes a concheros de origen antrópico de diferente cronología. En cada par seleccionado, una muestra representa el equilibrio con el reservorio atmosférico (carbón vegetal) y la otra con el reservorio marino (valva de molusco). Las muestras marinas analizadas corresponden a bivalvos meso $e$ infra litorales de la familia Mytilidae: Mytilus edulis (mejillón) y Aulacomya ater (cholga). Estos moluscos viven adheridos mediante un biso a las rocas de las plataformas de abrasión de ola o restingas, que poseen gran continuidad a lo largo de la costa norte del golfo San Matías. Las muestras terrestres corresponden a carbones procedentes de distintos arbustos del monte patagónico, que se utilizan como leña aún en la actualidad. Las muestras de cada par proceden de la misma unidad estratigráfica expuesta durante la excavación de los concheros, que procedió por niveles artificiales de $5 \mathrm{~cm}$ (ver Favier Dubois y Borella 2007).

Para el presente estudio fueron seleccionadas diez lentes de valvas correspondientes a seis localidades diferentes de la costa norte del golfo San Matías (Fig. 2). Las muestras arqueológicas fueron elegidas de acuerdo con los siguientes criterios: a) muestras procedentes de las localidades más antiguas a las más recientes, a fin de evaluar posibles cambios en el ERM a lo largo del tiempo; y b) muestras correspondientes a localidades de diferentes morfología a lo largo del sector septentrional del golfo, como caletas, bahías y playas a mar abierto. Esto a fin de evaluar posibles diferencias vinculadas al contacto restringido o no restringido del agua costera con el mar abierto.

Por otro lado, se tomaron muestras modernas del área a fin de estimar un valor actual de ERM. En este caso la muestra de origen marino correspondió a valvas aún articuladas de Mytilus edulis obtenidas de la línea de resaca de pleamar, y la de origen continental a semillas de un arbusto nativo, el alpataco (Prosopis flexuosa var. depressa), colectadas de una planta viva. Ambas muestras procedentes de la misma localidad, Barranca de los Concheros (Fig. 2).

Tanto los pares arqueológicos como el par moderno fueron datados por acelerador en el laboratorio de AMS de la Universidad de Arizona, USA (NSF Arizona AMS Laboratory) de acuerdo con rutinas establecidas.

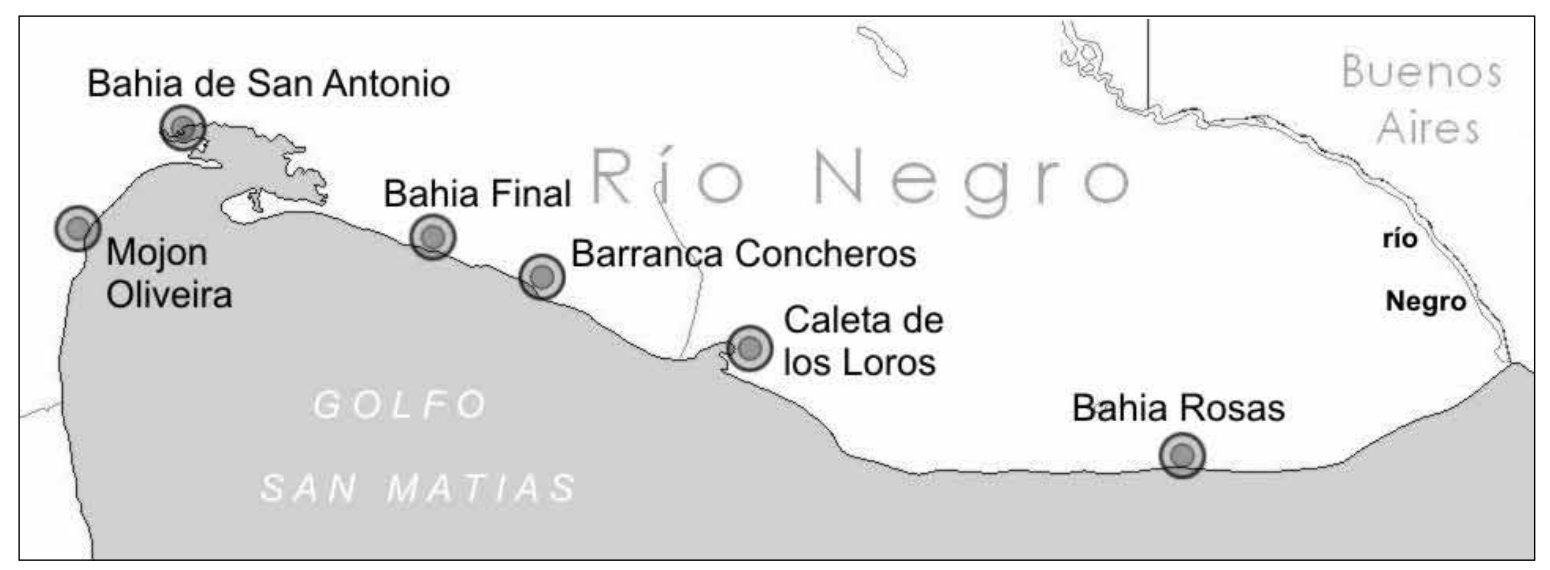

Fig. 2. Ubicación de las localidades arqueológicas seleccionadas para este estudio. 
Descripción de las localidades seleccionadas

A continuación se reseñan algunas de las características más relevantes de cada localidad muestreada en relación con los estudios de efecto reservorio (ver también Tabla 1 ):

a-Bahía Rosas (BR): es una bahía muy abierta ubicada a $40 \mathrm{~km}$ al sudoeste de la desembocadura del río Negro. Los concheros se localizan en dunas desarrolladas sobre cordones litorales que conforman una amplia terraza holocena. En la actualidad no se observan restingas en las playas de esta bahía, pero resulta indudable su presencia en el pasado, donde los grupos humanos obtuvieron mejillones y cholgas hace ca. 4.000 años AP (Favier Dubois et al. 2006). Los acantilados que bordean esta bahía, así como sus plataformas litorales, corresponden a la Fm. Río Negro (Plioceno), compuesta por areniscas y limolitas silicoclásticas de origen eólico y fluvial (Andreis 1965).

b-Caleta de los Loros (CL): constituye un entrante costero estrecho y profundo de dominio mareal (boca de mareas) al reparo de una espiga que ha ido creciendo a lo largo del Holoceno y tiende a cerrarlo (Del Río y Colado 1999). Los concheros se ubican en las dunas que se desarrollan alrededor de este entrante, sobre cordones litorales elevados. En el acantilado que lo flanquea al Oeste y plataformas de abrasión de ola aflora la Fm. Río Negro.

c-Barranca de los Concheros (BCH): es un sector acantilado sobre el que se elevan depósitos coluvio-eólicos que preservan un gran número de concheros separados verticalmente en estratigrafía. En un sector de $20 \mathrm{~m}$ de altura se muestrearon 16 lentes de valvas superpuestas. Cuatro de ellas fueron datadas para los estudios de ERM, incluyendo las del tope y base de la secuencia. El acantilado y la plataforma de abrasión de ola corresponden a la Fm. Patagonia (Mioceno) compuesta principalmente por limolitas amarillo verdosas con intercalaciones arcillosas de origen marino (Camacho 1967). Por lo general esta formación posee fósiles carbonáticos, siendo frecuente la presencia de grandes ejemplares de Ostrea sp. así como de gasterópodos y erizos.

d-Bahía Final (BF): es una bahía muy abierta en la que los concheros se ubican entre dunas sobre cordones litorales holocenos, a diferentes distancias de la playa actual. En BF6 se dataron dos concheros, uno próximo a la costa actual y el otro ubicado $380 \mathrm{~m}$ hacia el continente, sobre un paleoacantilado correspondiente al Holoceno medio, que resultó mucho más antiguo (Tabla 2). La plataforma de abrasión de ola corresponde a la Fm. Baliza San Matías (Pleistoceno), que consiste en un conglomerado de origen marino color castaño oscuro a castaño amarillento con rodados y valvas de moluscos enteras o fragmentadas (frecuentemente Amiantis purpurata y Mactra sp.), y matriz arenosa, fuertemente cementado (Angulo et al. 1978).

e-Bahía de San Antonio/San Antonio Oeste (SAO): esta amplia y profunda bahía constituye el principal accidente costero del litoral rionegrino. La localidad de San Antonio Oeste ocupa el sector occidental de la misma, y se asienta sobre una elevada terraza marina compuesta por cordones litorales (Angulo et al. 1978), cubierta de depósitos eólicos que registran la presencia de concheros. En las plataformas de abrasión de ola aflora la Fm. Baliza San Matías.

f-Mojón Oliveira: se localiza en un pequeño cabo que asoma en el acantilado desarrollado al sudoeste de la bahía de San Antonio. Sobre este

Tabla 1. Localidades muestreadas en la costa norte del golfo San Matías.

\begin{tabular}{|c|c|c|c|}
\hline Localidades & Lat/long. & Contexto de los concheros & Variables locales relevantes \\
\hline a. Bahía Rosas (BR) & $\begin{array}{l}41^{\circ} 09^{\prime \prime} \mathrm{S} \\
63^{\circ} 22 " \mathrm{~W}\end{array}$ & Dunas sobre terraza marina & $\begin{array}{l}\text { Mar abierto. Relativamente próxima a la } \\
\text { desembocadora del río Negro. }\end{array}$ \\
\hline b. Caleta de los Loros (CL) & $\begin{array}{l}41^{\circ} 01^{\prime \prime} \mathrm{S} \\
64^{\circ} 07^{\prime \prime} \mathrm{W}\end{array}$ & Dunas sobre terraza marina & $\begin{array}{l}\text { Caleta profunda. Conexión restringida } \\
\text { con el mar abierto. }\end{array}$ \\
\hline c. Barranca de los Concheros (BCH) & $\begin{array}{l}40^{\circ} 55^{\prime \prime} \mathrm{S} \\
64^{\circ} 23 " \mathrm{~W}\end{array}$ & $\begin{array}{l}\text { Depósitos coluviales } \\
\text { del paleoacantilado }\end{array}$ & $\begin{array}{l}\text { Mar abierto. Carbonatos fósiles en faja } \\
\text { intermareal. }\end{array}$ \\
\hline d. Bahía Final (BF) & $\begin{array}{l}40^{\circ} 52 " \mathrm{~S} \\
64^{\circ} 30^{\prime \prime} \mathrm{W}\end{array}$ & $\begin{array}{l}\text { Dunas sobre terraza marina } \\
\text { y paleoacant. }\end{array}$ & $\begin{array}{l}\text { Mar abierto. Carbonatos fósiles en faja } \\
\text { intermareal. }\end{array}$ \\
\hline e. Bahía de San Antonio (SAO-PC) & $\begin{array}{l}40^{\circ} 44^{\prime \prime} \mathrm{S} \\
64^{\circ} 57^{\prime \prime} \mathrm{W}\end{array}$ & Dunas sobre terraza marina & $\begin{array}{l}\text { Bahía de grandes dimensiones. } \\
\text { Conexión restringida con el mar abierto. }\end{array}$ \\
\hline f. Mojón Oliveira (MO) & $\begin{array}{l}41^{\circ} 09 " \mathrm{~S} \\
63^{\circ} 22 " \mathrm{~W}\end{array}$ & Dunas sobre el acantilado & $\begin{array}{l}\text { Mar abierto. Carbonatos fósiles en faja } \\
\text { intermareal. }\end{array}$ \\
\hline
\end{tabular}


cabo se emplazan dunas que contienen las lentes de valvas de origen arqueológico. El acantilado y plataforma de abrasión de ola corresponden a la Fm. Patagonia.

\section{RESULTADOS}

La datación de los pares arqueológicos proveyó veinte edades radiocarbónicas con las que se obtuvieron diez diferencias de edad entre las muestras marina y continental, presumiblemente referidas a efecto reservorio. El molusco del par moderno, no obstante, evidenció una actividad radiocarbónica mayor a moderna (aunque no especificada por el laboratorio), atribuible al "efecto bomba" de acuerdo al informe de datación radiocarbónica (muestra AA77307). Este efecto se debe a la radiación artificial inducida a la atmósfera a causa de los ensayos nucleares realizados en la década del 50 (de Vries 1958), aunque no puede descartarse algún otro tipo de contaminación radioactiva de esa muestra. Ello impidió obtener un valor de ERM actual en la zona de estudio.

Las diferencias de edad atribuibles al efecto reservorio marino en las muestras malacológicas no manifiestan en apariencia tendencias temporales.
Fluctúan a lo largo del período 5.300-700 años AP entre 205 y 358 años (Tabla 2 y Fig. 3). El valor promedio que proporcionan es de 266 años radiocarbónicos y el desvío estándar de 51 años.

\section{DISCUSIÓN}

Una lente de conchero puede resultar un contexto muy adecuado para estudios de efecto reservorio. La elevada tasa de descarte de los moluscos durante su consumo genera un depósito que posee una elevada resolución temporal, particularmente cuando las valvas no han sufrido procesos de dispersión, trituración y/o compactación. Para el caso de los concheros de la costa norte del golfo San Matías los estudios geoarqueológicos preliminares han mostrado una preservación excelente de las valvas en su matriz, y han permitido evaluar los procesos de formación involucrados, particularmente las propiedades espaciales y temporales de estos depósitos derivadas de las formas de consumo y descarte de las valvas (ver Favier Dubois y Borella 2007). De acuerdo con estos estudios y en forma similar a lo propuesto en trabajos previos (Deodat 1967; Caviglia y Borrero 1978; Orquera 1999; Gómez Otero et al. 1999) los moluscos colectados

Tabla 2. Valores de ERM en el golfo San Matías entre ca. 5.300 y 700 años AP.

\begin{tabular}{|c|c|c|c|c|c|}
\hline $\begin{array}{c}\text { Sitios } \\
\text { (de E a O) }\end{array}$ & Pares seleccionados & $\begin{array}{c}\text { Edades } \\
\text { AMS } \\
\text { (años AP) }\end{array}$ & $\delta^{13} \mathrm{C}$ & $\begin{array}{l}\text { № Lab. } \\
\text { (Arizona) }\end{array}$ & $\begin{array}{c}\text { Diferencia de } \\
\text { edades }^{1}\end{array}$ \\
\hline 1. BR2 & $\begin{array}{l}\text { Carbón } \\
\text { Valva (Mytilus) }\end{array}$ & $\begin{array}{l}3.985 \pm 41 \\
4.250 \pm 41\end{array}$ & $\begin{array}{c}-25.4 \\
3.1\end{array}$ & $\begin{array}{l}\text { AA77298 } \\
\text { AA77297 }\end{array}$ & 265 \\
\hline 2. CL3 & $\begin{array}{l}\text { Carbón } \\
\text { Valva (Mytilus) }\end{array}$ & $\begin{array}{l}2.108 \pm 35 \\
2.346 \pm 35\end{array}$ & $\begin{array}{c}-23.6 \\
2.1\end{array}$ & $\begin{array}{l}\text { AA77300 } \\
\text { AA77299 }\end{array}$ & 238 \\
\hline 3. $\mathrm{BCH} 1$ & $\begin{array}{l}\text { Carbón } \\
\text { Valva (Aulacomya) }\end{array}$ & $\begin{array}{l}2.839 \pm 42 \\
3.142 \pm 53\end{array}$ & $\begin{array}{c}-10.4 \\
3.1 \\
\end{array}$ & $\begin{array}{l}\text { AA64774 } \\
\text { AA74744 }\end{array}$ & 303 \\
\hline 4. $\mathrm{BCH} 4$ & $\begin{array}{l}\text { Carbón } \\
\text { Valva (Mytilus) }\end{array}$ & $\begin{array}{l}2.984 \pm 50 \\
3.230 \pm 52\end{array}$ & $\begin{array}{c}-22.7 \\
2.2 \\
\end{array}$ & $\begin{array}{l}\text { AA74746 } \\
\text { AA74745 }\end{array}$ & 246 \\
\hline 5. $\mathrm{BCH} 10$ & $\begin{array}{l}\text { Carbón } \\
\text { Valva (Aulacomya) }\end{array}$ & $\begin{array}{l}2.482 \pm 49 \\
2.708 \pm 61\end{array}$ & $\begin{array}{c}-15.4 \\
1.5\end{array}$ & $\begin{array}{l}\text { AA74748 } \\
\text { AA74747 }\end{array}$ & 226 \\
\hline 6. $\mathrm{BCH} 16$ & $\begin{array}{l}\text { Carbón } \\
\text { Valva (Mytilus) }\end{array}$ & $\begin{array}{l}1.772 \pm 36 \\
2.106 \pm 54\end{array}$ & $\begin{array}{l}-10 \\
1.7 \\
\end{array}$ & $\begin{array}{l}\text { AA64775 } \\
\text { AA74749 } \\
\end{array}$ & 334 \\
\hline 7. BF6 costa & $\begin{array}{l}\text { Carbón } \\
\text { Valva (Mytilus) }\end{array}$ & $\begin{array}{c}740 \pm 40 \\
1.009 \pm 59\end{array}$ & $\begin{array}{c}-24.3 \\
1.3\end{array}$ & $\begin{array}{l}\text { AA64772 } \\
\text { AA74750 }\end{array}$ & 269 \\
\hline 8. BF6 Paleoac. & $\begin{array}{l}\text { Carbón } \\
\text { Valva (Aulacomya) }\end{array}$ & $\begin{array}{l}3.430 \pm 43 \\
3.643 \pm 56 \\
\end{array}$ & $\begin{array}{c}-21.7 \\
2.9 \\
\end{array}$ & $\begin{array}{l}\text { AA64773 } \\
\text { AA74751 }\end{array}$ & 213 \\
\hline 9. $\mathrm{SAO}-\mathrm{PC}$ & $\begin{array}{l}\text { Carbón } \\
\text { Valva (Mytilus) }\end{array}$ & $\begin{array}{l}5.290 \pm 39 \\
5.648 \pm 40\end{array}$ & $\begin{array}{c}-11.2 \\
1.7\end{array}$ & $\begin{array}{l}\text { AA77304 } \\
\text { AA77303 }\end{array}$ & 358 \\
\hline 10. MO-1 & $\begin{array}{l}\text { Carbón } \\
\text { Valva (Aulacomya) }\end{array}$ & $\begin{array}{l}715 \pm 33 \\
920 \pm 35 \\
\end{array}$ & $\begin{array}{c}-24.2 \\
3.1 \\
\end{array}$ & $\begin{array}{l}\text { AA77306 } \\
\text { AA77305 } \\
\end{array}$ & 205 \\
\hline \multicolumn{5}{|c|}{ Promedio y desvío } & 266 +51 años \\
\hline
\end{tabular}

${ }^{1}$ Calculadas considerando el valor medio de la edad informada. 


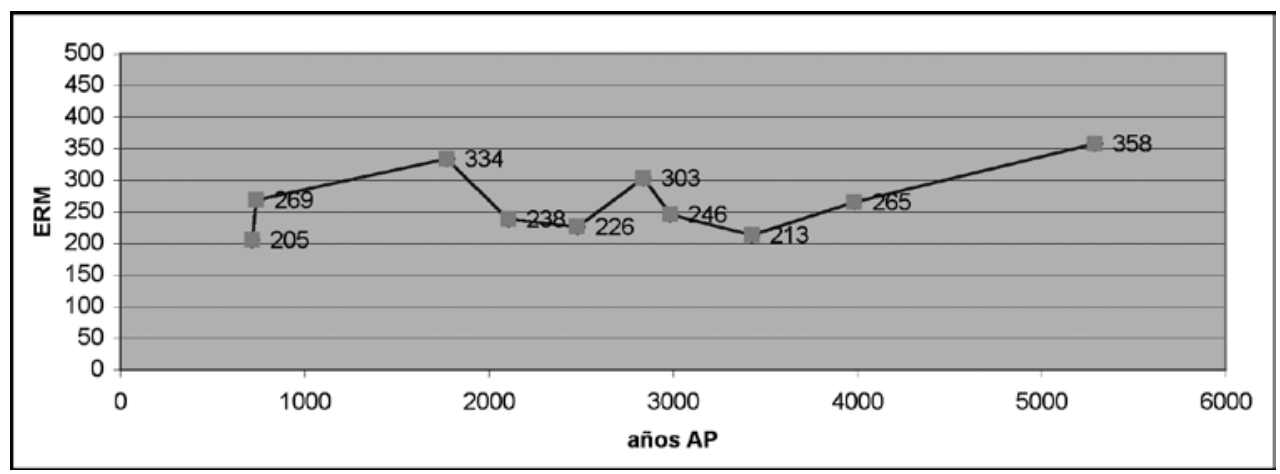

Fig. 3. Fluctuación en los valores de ERM obtenidos en el intervalo ca. 700-5.300 años AP.

habrían sido arrojados sobre pequeños fogones a fin de abrir las firmemente cerradas valvas, y consumidos inmediatamente, descartándose las conchas en el mismo lugar. El fuego se habría mantenido activo agregando nuevas ramas hasta procesar la cantidad de valvas deseada. Tal dinámica permitiría asumir simultaneidad en la depositación de los carboncitos del fogón y de las valvas chamuscadas que los rodean que, en conjunto, constituyen la lente del conchero. Con posterioridad, estas acumulaciones se vieron sepultadas por arena eólica, hecho que ha facilitado su excelente preservación y colaborado en garantizar una elevada resolución e integridad del material contenido en ellas (Favier Dubois y Borella 2007).

Una vez evaluada la contemporaneidad entre las muestras marina y continental, otra variable importante a tener en cuenta para la estimación del efecto reservorio es la edad de cada una de ellas al incorporarse al conchero, esto es la edad del material. En el caso de los mejillones (Mytilus edulis), éstos alcanzan un tamaño de $4 \mathrm{~cm}$ (el mínimo para ser colectados para consumo) en unos 2 años, y llegan a vivir sólo unos 7 años (Trancart 1978). La cholga (Aulacomya ater) puede alcanzar esa talla más rápido (Solís y Lozada 1971) resultando poco más longeva que el mejillón. Esto provee una estimación de un lapso no mayor a una década para la edad introducida por la muestra marina.

Los arbustos utilizados como leña en los fogones, sin embargo, corresponden a especies de distinta longevidad. De acuerdo con los estudios antracológicos realizados en 8 concheros de esta misma área (Ortega y Marconetto 2008), los géneros utilizados como combustible fueron varios: Prosopis sp., Schinus sp., Bougainvillea sp., Larrea sp., Atriplex sp., Condalia sp. y Suaeda sp..
Algunos de ellos como Prosopis, Schinus o Condalia pueden vivir hasta unos 100 años (CONABIO 2001, Lamberto com pers. 2008). Sin embargo, es probable que las partes utilizadas como combustible en estos casos no hayan sido gruesos troncos sino ramas de menor diámetro, dado que lo que se pretendía no era un fuego importante o muy duradero sino el suficiente para abrir las valvas, sin calcinarlas. Esto reduciría el efecto de edad del material introducido por las especies longevas. Algunos de los carbones recuperados en los concheros han sido identificados como Atriplex sp., que correspondería a Atriplex lampa (zampa), un arbusto de ciclo anual (Cabrera 1970). Es interesante destacar que este arbusto es una planta $\mathrm{C} 4$ (posee valores enriquecidos en $\delta^{13} \mathrm{C}$ ), lo que lo diferencia de otros arbustos del monte patagónico, que en su mayoría son plantas C3. En el área bajo estudio, los arbustos $\mathrm{C} 4$ se hallan representados por esta especie y por Suaeda divaricata (jume) de mayor porte y más longevo. Ejemplares actuales de estas especies colectados en la zona han proporcionado valores de $\delta^{13} \mathrm{C}$ de -13.3\% y $-11.3 \%$ (Laboratorio de Isótopos Estables, INGEIS, Argentina). Si observamos ahora la Tabla 2, veremos que las diferencias mayores entre las edades obtenidas en cada uno de los diez pares analizados corresponden a aquellas en las que los carbones poseen valores de $\delta^{13} \mathrm{C}$ entre -10 y -11.2 $\%$, es decir que provienen de plantas C4. Esto parece tener sentido si se ha utilizado una planta anual como el Atriplex, que no agrega su edad al fechado y por tanto no reduce la diferencia entre las edades de cada reservorio, como pudo ocurrir con el uso de arbustos más longevos. Tal circunstancia parece verse particularmente ilustrada en el caso de las muestras de la localidad Barranca de los Concheros $(\mathrm{BCH})$. Allí se observa que los concheros superpuestos 1 y 
4, pese a tener la misma cronología, presentan una diferencia de valores de efecto reservorio de 57 años, evidenciando los carbones corresponder precisamente a un arbusto $\mathrm{C} 4$ en el primer caso $\left(\delta^{13} \mathrm{C}\right.$ : $\left.-10.4 \% 0\right)$ y a uno $\mathrm{C} 3$ en el segundo $\left(\delta^{13} \mathrm{C}\right.$ : $-22.7 \%$ ). Ello sugiere que puede considerarse algún efecto de edad del material en los valores obtenidos sobre carbones por este estudio, al menos en algunos casos. Se podría pensar entonces que el ERM más ajustado sería el promedio de las muestras que involucran solamente plantas C4 (muestras 3, 6 y 9 por ejemplo, con valores algo superiores a 300 años). Sin embargo, no se han identificado las especies de los carbones datados de la Tabla 2 (los estudios antracológicos fueron posteriores), y podrían hallarse involucrados otros factores en estas diferencias de edad, por lo que se considera al momento más prudente mantener como valor de referencia el promedio de todos los pares.

En relación con otra variable que puede afectar los valores de ERM, como es la morfología costera, las localidades ubicadas frente a masas de agua con conexión restringida con el mar abierto -como caleta de los Loros y bahía de San Antonio- no han manifestado una tendencia a disminuir sus valores en relación a aquellos obtenidos a mar abierto. Es decir, no muestran edades aparentes que puedan interpretarse como un mayor equilibrio con el reservorio atmosférico generado por esta restricción. En este sentido tampoco parece importante la influencia del aporte de aguas continentales, cuyas especies de carbono inorgánico disuelto $\left(\mathrm{CO}_{2}\right.$, bicarbonatos y carbonatos) se hallarían en equilibrio con la atmósfera y tenderían a deprimir el ERM (Figini 1999). El único río permanente que podría generar tal efecto es el propio río Negro (ver Fig. 2), ya que los otros cursos presentes en esta región semiárida son arroyos temporarios, cuando no cárcavas, sólo activas durante episodios de lluvias torrenciales. Este importante río patagónico ejerce influencia desde su desembocadura, y hacia donde son arrastradas sus aguas por deriva litoral. Sin embargo, la localidad más próxima a su boca, Bahía Rosas, no llega a manifestar una evidente depresión en su edad aparente, que es de 265 años.

Por último, la presencia de carbonatos envejecidos en las restingas o plataformas de abrasión de ola donde se fijan los mitílidos daría lugar, por el contrario, a un incremento en las diferencias de edad. No obstante, tampoco resulta claramente expresado este efecto en los casos en que la geología indica la presencia de fósiles carbonáticos, como es el caso en Barranca de los Concheros (edades aparentes de 303, 246, 226 y 334 años), Bahía Final (edades aparentes de 269 y 213 años), o Mojón Oliveira (205 años), donde los valores se ubican tanto por debajo como por encima de la media obtenida.

\section{CONCLUSIONES}

La evaluación del ERM requiere que las muestras sean contemporáneas y que no existan diferencias en cuanto a edad del material entre ellas, o que éstas sean mínimas. En los casos arqueológicos estos requisitos pueden ser difíciles de lograr. Sin embargo el análisis de los contextos de asociación y de la longevidad de las especies involucradas permite una primera aproximación valiosa. Por otro lado, desglosar algún efecto local en particular no es sencillo, siendo posible que se conjuguen distintos factores en forma simultánea influyendo en las diferencias de edad obtenidas. Tampoco puede desestimarse alguna fluctuación temporal en los valores de ERM (ver Deo et al. 2004). Sin embargo, consideramos que los resultados de este estudio permiten acotar, al menos preliminarmente, su rango de variación a la escala espacial y temporal considerada.

La corrección por ERM se halla estandarizada en 400 años de acuerdo con el promedio oceánico global (Stuiver y Braziunas 1993), este valor es introducido por los programas de calibración de no disponerse de otro más ajustado. Sin embargo, la costa atlántica patagónico-fueguina, en sus más de 3.000 $\mathrm{km}$ de extensión, presenta un significativo espectro de valores, en parte derivados del efecto latitudinal pero primordialmente de efectos locales. Los estudios previos de ERM actual realizados sobre 20 pares de muestras de moluscos y plantas colectados en Patagonia y Tierra del Fuego mostraron diferencias de edades ${ }^{14} \mathrm{C}$ entre 80 y 800 años, que ilustran claramente la influencia de éstos últimos (ver Cordero et al. 2003). Ello pone en relieve la importancia de realizar estudios de efecto reservorio en cada área bajo investigación para contar con valores más representativos.

El valor medio de ERM obtenido en este estudio (266 \pm 51 años) representa un valor de corrección sensiblemente menor al promedio oceánico global, permitiendo un ajuste temporal más adecuado para las muestras de moluscos (mitílidos) datadas 
por ${ }^{14} \mathrm{C}$ en la costa norte del golfo San Matías. En este caso, el peso de los factores locales no parece ser tan marcado como en otros sectores del litoral patagónico, por lo que su diferencia con el valor proporcionado por los mejillones en el Canal de Beagle (556+61 años) atestigua el efecto latitudinal existente entre los organismos colectados en Norpatagonia (40\%/41 latitud S) y en el sur de Tierra del Fuego (54º latitud S).

La determinación de un valor de ERM local (aunque siempre aproximado) se hace particularmente útil para las ocupaciones humanas correspondientes al Holoceno medio y tardío, donde diferencias de centurias respecto al valor oceánico global de corrección pueden resultar importantes en la interpretación de la cronología cultural. Esto es aún más importante para la arqueología de Patagonia, en donde las poblaciones humanas mantuvieron un estilo de vida cazador recolector hasta el contacto europeo, y particularmente para áreas costeras donde las valvas de moluscos constituyen uno de los materiales orgánicos mayormente representados (en ocasiones el único) en el registro arqueológico.

\section{AGRADECIMIENTOS}

Estas investigaciones fueron financiadas por los proyectos PIP CONICET 6415, National Geographic Society CRE Grant 8171-07 y PICT Agencia 38264. Agradezco especialmente al Dr. Timothy Jull (NSF Arizona AMS Laboratory) por su gentileza en hacernos un descuento para las dataciones por AMS para este estudio. Asimismo al Consejo de Ecología y Medio Ambiente de la Provincia de Río Negro (CODEMA) por autorizarnos a trabajar en áreas protegidas. También expreso mi agradecimiento a los evaluadores del trabajo cuyos comentarios y sugerencias han sido muy útiles para mejorar el manuscrito, así como al Dr. Enrique Morsan por los datos aportados. Este estudio fue posible gracias a la participación de todos los integrantes del equipo de investigación que colaboraron en distintos aspectos.

\section{BIBLIOGRAFÍA}

ALBERO M., ANGIOLINI F. E., Y PIANA E. L. 1986. Discordant Ages Related to Reservoir Effect of Associated Archaeologic Remains from the Tunel Site, Beagle Channel, Argentine Republic. Radiocarbon 28 (2A): 748-753.
ALBERO M., ANGIOLINI F. E., Y PIANA E. L. 1987. Holocene $14 \mathrm{C}$ reservoir effect at Beagle Channel (Tierra del Fuego, Argentina Republic), Quaternary of South America and Antartic Peninsula 5: 59-71.

ANDREIS, R. R. 1965. Petrografía y paleocorrientes de la Formación Río Negro (tramo General Conesa-Boca del Río Negro), Revista Museo de La Plata, Geología 36: 245-310, La Plata.

ANGULO, R., F. FIDALGO, M. GÓMEZ DEL PERAL y E. SCHNACK. 1978. Las ingresiones marinas Cuaternarias en la bahía de San Antonio y sus vecindades. VII Congreso Geológico Argentino, Actas (1): 271-283, Neuquen.

BRAN, D., G. CECCHI Y A. BALMACEDA y LORES. 1985. Vegetación. En: Relevamiento Integrado de los Recursos Naturales de la Provincia de Río Negro, Convenio INTA- Ministerio de Recursos Naturales (Pcia de Río Negro). MS.

CABRERA A. L. 1970. Flora de la Provincia de Buenos Aires. Fundación Buen Aire.

CABRERA, A. L. y A. WILLINK. 1980. Biogeografía de América Latina. Monografías de la OEA, Washington D.C.

CAMACHO H. H. 1967. Las transgresiones del Cretácico superior y Terciario de la Argentina. Revista de la Asociación Geológica Argentina 22 (4): 253-280, Buenos Aires.

CAVIGLIA, S. E. y L. A. BORRERO. 1978. Bahía Solano: su interpretación paleoetnozoológica en un marco regional. Actas V Congreso Nacional de Arqueología Argentina, San Juan. MS.

CONABIO 2001. Comisión Nacional para el conocimiento y uso de la Biodiversidad (México). Sitio http://www. conabio.gob.mx/malezasdemexico/biologíayecología.

CORDERO OTERO R., FAVIER DUBOIS C. M. y PANARELLO H. 1999. Efecto reservorio en las dataciones por ${ }^{14} \mathrm{C}$ sobre moluscos actuales de la costa patagónico-fueguina. Importancia arqueológica. En: Desde el País de los Gigantes. Perspectivas arqueológicas en Patagonia II: 541-546, Río Gallegos.

CORDERO OTERO R., H. PANARELLO, S. LANZELOTTI y FAVIER DUBOIS C. M. 2003. Radiocarbon age offsets between living organisms from the marine and continental reservoir in coastal localities of Patagonia (Argentina). Radiocarbon 45 (1): 9-15.

DEL RÍO, J. L. y U. R. COLADO. 1999. Ambientes sedimentarios actuales de la Caleta de los Loros, Provincia de Río Negro, República Argentina. Thalassas 15: 35-44

DEO, J. N., J. O. STONE y J. K. STEIN. 2004. Building confidence in shell: variations in the marine radiocarbon reservoir correction for the Northwest Coast over the past 3000 years, American Antiquity 69: 771-786. 
DEODAT, L. S. 1967. Una antigua manufactura Valvácea en el Golfo de San Matías (Argentina). Runa X(1-2):319-353. Buenos Aires.

DE VRIES H. 1958. Atom Bomb Effect: variations of radiocarbon in plants, shells, snails in the past 4 years. Science 128: 250-251.

FAVIER DUBOIS C. M. y F. BORELLA. 2007. Consideraciones acerca de los procesos de formación de concheros en la costa Norte del golfo San Matías (Río Negro, Argentina). Cazadores- Recolectores del Cono Sur 2: 151-165.

FAVIER DUBOIS C. M., F. BORELLA, L. MANZI, M. CARDILLO, S. LANZELOTTI, F. SCARTASCINI, C. MARIANO y E. BORGES VAZ. 2006. Aproximación regional al registro arqueológico de la costa rionegrina. En: Arqueología de la Costa Patagónica, perspectivas para la conservación. Editado por I. Cruz y S. Caracotche, Capítulo 3, pp. 50-68. UNPA, Río Gallegos.

FIGINI, A. J. 1999. Comparación de Edades C-14 en Muestras de Origen Marino y Terrestre. Efecto de Reservorio. Actas del XII Congreso Nacional de Arqueología Argentina, Tomo II: 353-356. La Plata.

GÓMEZ OTERO J., J. B BELARDI, A. SÚNICO y R. TAYLOR. 1999. Arqueología de Cazadores-recolectores en Península Valdés (costa central de Patagonia): primeros resultados. Soplando en el viento... Actas de las Terceras Jornadas de Arqueología de la Patagonia, pp. 393-417. Neuquén y Buenos Aires.
KENNETT D. J., B. L. INGRAM, J. M. ERLANDSON y P. WALKER. 1997. Evidences for temporal fluctuations in marine radiocarbon reservoir ages in the Santa Barbara Channel, Southern California. Journal of Archaeological Science 24: 1051-1059.

OLSSON, I.U. 1983. Dating non-terrestrial materials. Proceedings $1^{\text {st }}$ International Symposium C-14 and Archaeology, Mook W. y Waterbolk H., eds. P. act 8: 277-294.

ORQUERA, L. A. 1999. El consumo de moluscos por los canoeros del extremo sur. Relaciones de la Sociedad Argentina de Antropología XXIV:307-327.Buenos Aires.

ORTEGA F. y M. B. MARCONETTO. 2008. Una discusión "encendida" primeros resultados de los análisis de vestigios de combustión en concheros de la costa norpatagónica (Río Negro). En: Arqueología de Patagonia: una mirada desde el último confín, editado por M. Salemme, F. Santiago, M. Alvarez, E. Piana, M. Vázquez y M. E. Mansur, Editorial Utopías, Ushuaia (en prensa).

SOLIS I. y E. LOZADA. 1971. Algunos aspectos biológicos de la cholga de Magallanes Aulacomya ater Mol. Biol. Pesq. Chile Nro. 5 pp. 109-144. Santiago de Chile.

STUIVER M. y T. F. BRAZIUNAS. 1993. Modeling Atmospheric 14C Influences and 14C Ages of Marine Samples to 10.000 BC, Radiocarbon 35 (1): 137-189.

TRANCART M. 1978. Biologie et possibilities d'exploitation d'Mytilus platensis dans le golfe San Jose, Península Valdes. Doctorat 3eme cycle Oceanologie, 87 p. 
